\title{
ACS/TIRVIM: Calibration and first results
}

Alexey Shakun, Nikolay Ignatiev, Michail Luginin, Alexey Grigoriev, Boris Moshkin, Davide Grassi, Gabriele Arnold, Alessandro Maturilli, Andrey Kungurov, Vladislav Makarov, Fedor Martynovich, Igor Maslov, Dmitry Merzlyakov, Yuri Nikolskiy, Dmitry Patsaev, Aleksandr Santos-Skripko, Oleg

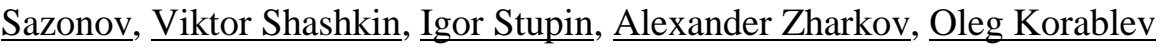

\author{
Alexey Shakun , Nikolay Ignatiev, Michail Luginin , Alexey Grigoriev , Boris Moshkin \\ Space Research Institute (Russian Federation); Davide Grassi Istituto di Astrofisica e Planetologia Spaziali \\ (IAPS-INAF) (Italy), Gabriele Arnold, Alessandro Maturilli Deutsches Zentrum für Luft- und Raumfahrt e.V. \\ (Germany); Andrey Kungurov, Vladislav Makarov, Fedor Martynovich , Igor Maslov, Dmitry Merzlyakov, \\ Yuri Nikolskiy, Dmitry Patsaev , Aleksandr Santos-Skripko , Oleg Sazonov, \\ Viktor Shashkin, Igor Stupin , Alexander Zharkov, Oleg Korablev \\ Space Research Institute (Russian Federation)
}

Proceedings Volume 10765, Infrared Remote Sensing and Instrumentation XXVI; 107650E (2018) https://doi.org/10.1117/12.2322163

\begin{abstract}
ABSRACT
Atmospheric Chemistry Suite (ACS) is a part of Russian contribution to ExoMars Trace Gas Orbiter (TGO) ESA-Roscosmos mission. ACS includes three separate infrared spectrometers (MIR, NIR and TIRVIM) with a different spectral coverage and targeted to the different science goals. ACS TIRVIM is a Fourier-transform spectrometer based on 2-inch double pendulum interferometer. It operates in the spectral range of 1.7-7 $\mu \mathrm{m}$ with the best spectral resolution $0.13 \mathrm{~cm}^{-1}$ for solar occultation (SO) mode and $0.8 \mathrm{~cm}^{-1}$ for nadir mode. In nadir mode TIRVIM is purposed to thermal sounding of the Martian atmosphere and aerosol properties retrieval. In SO mode TIRVIM is dedicated to trace gases measurements complementing to ACS MIR. After successful launch of ExoMars TGO on 16 April 2016 there were three time slots for turning on science instruments during cruise phase to execute necessary checks and calibration measurements. In March 2018 the nominal science orbit was reached after cruise and aerobraking phases. The first results of TIRVIM data processing show high performance of the instrument.
\end{abstract}

\section{INTRODUCTION}

The ExoMars TGO is joint ESA-Roscosmos mission to Mars. A key goal of the mission is studying of trace gases (particularly methane) in Martian atmosphere which could be a marker of biological or geological activity. Another mission goal is long term monitoring of the atmospheric composition and temperature [1]. The TGO payload includes four instruments: ACS (three infrared spectrometers); CASSIS (high-resolution color stereo camera); FREND (neutron detector) and NOMAD (ultraviolet and two infrared spectrometers).

ACS consists of three infrared channels: near-infrared (NIR); mid-infrared (MIR) and thermal infrared (TIRVIM). NIR is echelle spectrometer with acousto-optic tunable filter (AOTF) for diffraction orders selection. It covers spectral range $0.73-1.7 \mu \mathrm{m}$ with spectral resolving power up to 27000 . NIR is able to operate in nadir and occultation modes. Instrument is targeted to measurements of water vapor, aerosols and airglows (day and night). MIR is cross dispersion spectrometer for spectral range 2.3-4.3 
$\mu \mathrm{m}$ and spectral resolving power up to 50000. It operates only in occultation mode and aimed on trace gases detection [2].

TIRVIM is a Fourier-transform spectrometer (FTS) based on 2-inch double pendulum interferometer. It operates in 1.7-17 $\mu \mathrm{m}$ spectral range, which limited by the transparency of the optics and detector spectral sensitivity. The PV-MCT detector $(1.5 \mathrm{~mm}$ in diameter) cooled to cryogenic temperature by Stirling cooler is applied. The one-side maximal optical path difference (MOPD) is $5 \mathrm{~cm}$ which corresponds to best spectral resolution of $0.13 \mathrm{~cm}-1$. There are two operation modes possible: occultation, with OPD of $5 \mathrm{~cm}$ and corresponding resolution $0.13 \mathrm{~cm}-1$ in retrieved spectrum; nadir, with OPD about $1 \mathrm{~cm}$ and corresponding resolution $1 \mathrm{~cm}-1$ in retrieved spectrum. Nadir mode applies for thermal sounding, occultation mode - for trace gases. It is also possible to operate with occultation orientation but with nadir resolution, for aerosol profiles retrieval (climatology mode). The TIRVIM abbreviation honors Prof Vassilii Ivanovich Moroz, the IR astronomer and the leader of planetary school in IKI during 1968-2004 [2, 3$]$.

This paper performs the TIR VIM calibration results based on laboratory and inflight measurements. The examples of thermal field and aerosol properties profiles retrieved from TIRVIM spectra are presented.

\section{ACS TIRVIM CALIBRATION}

Laboratory measurements with TIRVIM were mostly dedicated to radiometric calibrations in the thermo-vacuum chamber. Also observations of the Sun, measurements of a laser emission line, and measurements of transmission of a gas cell filled with nitrogen-methane mixture were exercised. Finally, the angular diameter of the field of view was characterized. Emissivity of the internal calibration black body was measured in DLR with its spare model identical to the flight one (Fig. 1).

Measured emissivity of the internal black body.

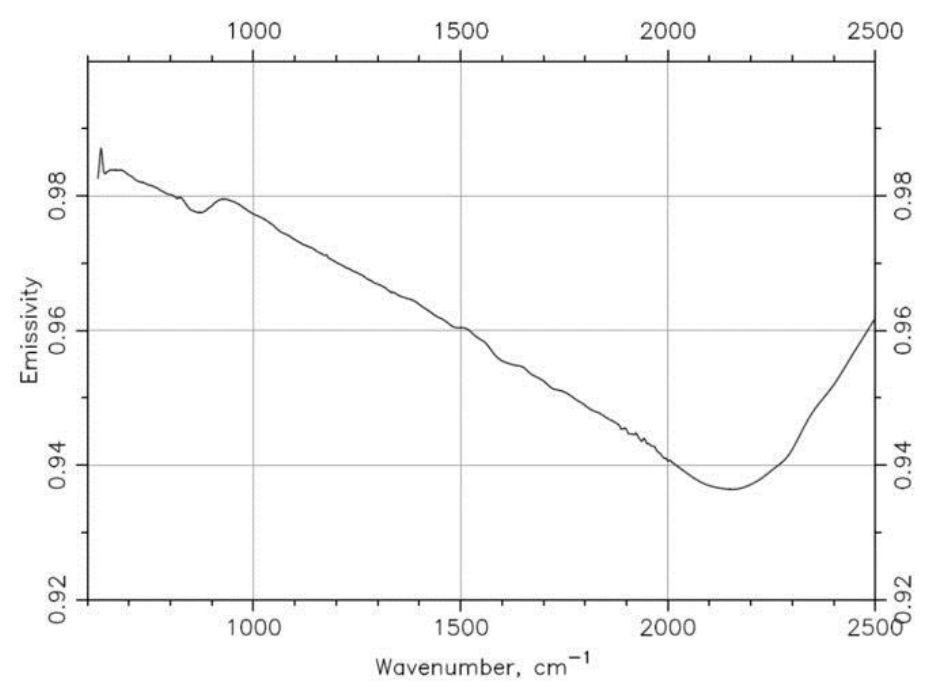

In flight calibrations include routine radiometric calibrations of the thermal IR part of the spectrum in the nadir mode and the field-of-view measurements of both nadir and occultation channels by means of the sun scanning. 
A quantity directly measured by a Fourier-transform spectrometer is the interferogram, the autocorrelation function of the electro-magnetic field [4]. Fourier transform of the interferogram gives the uncalibrated spectrum, which is generally a complex value given by a spectrum module and phase of the instrument. The radiometric calibration is a conversion of the interferogram or, equivalently, the uncalibrated complex spectrum to the spectral radiance. The calibrated radiance of any target can be derived from measurements of two objects with known spectral brightness [5]. In flight, the calibration sources are the cold space and an internal black body imitator with known spectral emissivity and controlled temperature, which are observed periodically during the measurements, by means of the scanner system. A simple calibration approach [5] operating with spectrum modules has been successfully applied to a number of space-born Fourier-transform spectrometers [6, 7, 8]. However, it appeared that TIRVIM is characterized by a source-dependent response, which requires a more complex approach to calibration operating with the complex value of the spectrum [9].

Mars nadir observations were carried out in two modes: with and without onboard interferogram averaging. The interferogram is taken with the reference channel frequency $f_{\mathrm{rc}}$. Originally, the Martian nadir observation mode was designed with $f_{\mathrm{rc}}=10.5-11.55 \mathrm{kHz}$. It appeared that in this regime the spectra of a constant source are slightly unstable with unknown reason. However, such instability was not observed in the regime designed for the solar occultation observation with $f_{\text {rc }}=71.1-79.0 \mathrm{kHz}$. The price for the higher $f_{\mathrm{rc}}$ is the increase of noise and informativity of the instrument. To achieve a similar signal to noise ratio an onboard interferogram averaging regime was implemented. In particular, averaging by 8 interferograms provides an acceptable signal to noise ratio. The averaging was switched off at the first week of operation in the nominal science orbit in March 2018. Then it was switched on till August 2018. After analysis of the measurements done in these months, the averaging mode appeared to be a cause of small and large errors in a considerable part of measurements and is supposed to be not used in the future for the following reason.

It may be easily shown that a necessary requirement for the averaging of interferograms is their alignment. Under stable conditions, interferograms are digitized at the same optical path difference points, although unknown: equal point numbers in a sequence of recorded interferograms do not correspond to equal path differences. In practice the interferograms are aligned according to their maximum and minimum values, in particular, the TIRVIM onboard software was designed in this way. With stable sources with the effective temperature sufficiently different from that of the instrument to provide a strong signal well above the noise level, like an internal calibration black body or a cold space, interferograms are stable and keep their shape. If a source slightly changes, the interferogram also changes, but positions of maximum and minimum in a path difference scale is not changed. For such sources, interferogram averaging can be easily done with the simple maximum or minimum alignment. However, in the presence of noise and a stronger variability of the source radiation, the interferogram maximum position can jump by one or more points, so that the maxima of the two adjacent interferograms do not correspond to the same path difference. Therefore, such interferograms require a more complex alignment and cannot be easily averaged by means of the actual onboard software. If anyway averaging is performed, spectra obtained from such interferograms are distorted. Such distortions are often easy to distinguish, but sometimes they are small in absolute values, but significant for scientific results: retrieved temperatures and especially aerosol opacity.

The sensitivity of the instrument and its noise level are characterized by the noise equivalent spectral radiance, which is determined as a standard deviation of the set of measurements of the same target 
(Fig.2). In our calibration method it can be also evaluated from the imaginary part of the calibrated spectrum, which must be equal to zero in the absence of noise [9]. A signal-to-noise ratio reaches its maximum at $700-800 \mathrm{~cm}^{-1}$ : on the dayside of Mars at warmest conditions it amounts to approximately 1500. Spikes centered at 920,1620 , and $1800 \mathrm{~cm}^{-1}$ are caused by the disturbances generated by the Stirling cryocooler.

Noise equivalent spectral radiance measured in regimes without interferogram averaging and with averaging by 8 interferograms.

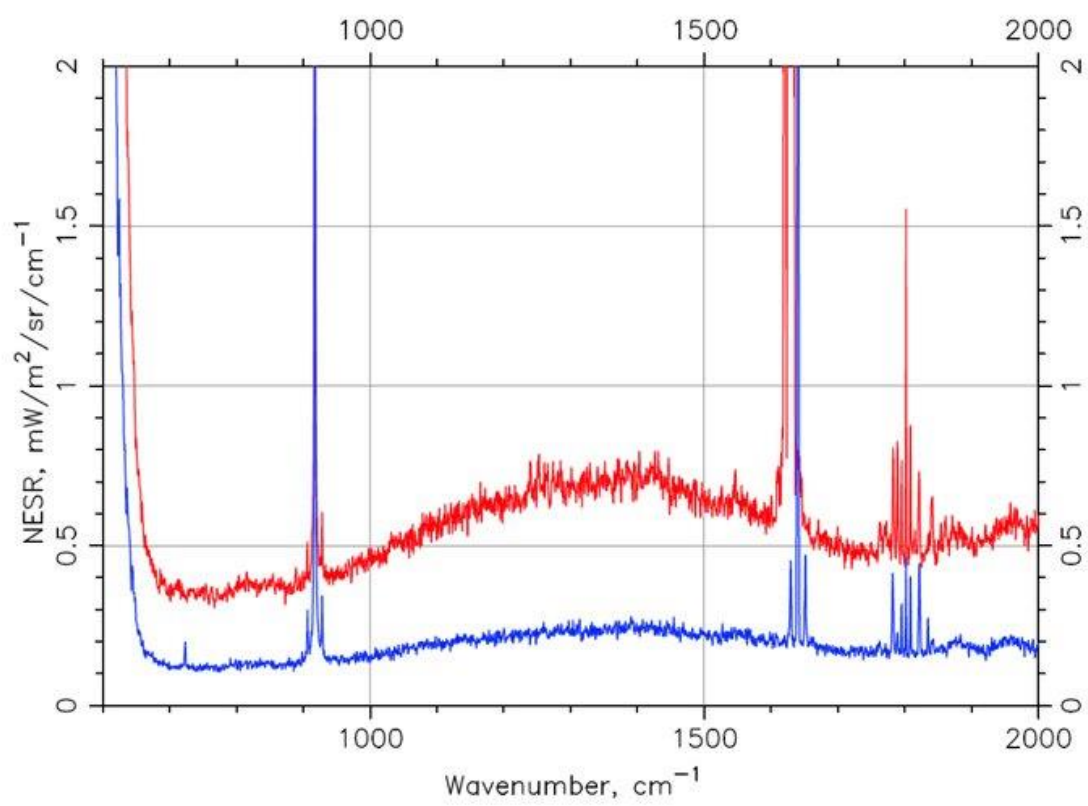

The black body emission at maximum source temperature of about $300 \mathrm{~K}$ becomes too low for the given sensitivity of the detector already at $2000 \mathrm{~cm}^{-1}$, therefore the radiometric calibration is valid only below this wavenumber. The short wavelength part of the TIRVIM spectral range $\left(2000-5500 \mathrm{~cm}^{-1}\right)$ is dedicated to observations in the solar occultation mode, which does not require absolute radiometric calibrations. The sensitivity in this part of the spectrum is too low for nadir observations.

\section{THERMAL SOUNDING RESULTS}

Thermal sounding in the $\mathrm{CO}_{2} 15 \mu \mathrm{m}$ band has been successfully carried out in a number of previous spaceborne experiments [e.g. 10, 11, 12, 13, 14]. The increased spectral resolution with respect to the previous measurements slightly improves both the altitude range (from the surface to 50-55 km) and the vertical resolution of the retrieved temperature profiles, which varies from several kilometers near the surface to $20 \mathrm{~km}$ high altitudes. Silicate dust and water ice absorption bands at 9 and $12 \mu \mathrm{m}$, respectively, are used to monitor simultaneously their optical depths. Temperature, dust and ice optical depth retrieval methods and algorithms are based on those developed for the PFS/Mars Express experiment [15]. Examples of measured calibrated spectra from a single orbit in the beginning of the nominal science phase in March, 2018, track of the field-of-view on the surface, and temperature field along the track are shown in Figs. 3- $\underline{5}$. To increase signal to noise ratio, spectra measured in the regime without interferogram averaging are to be averaged for a further processing.

Spectra of Mars of March 15, 2018: 42 (about 1\% of the total number) spectra from a single orbit distributed uniformly along the orbit, single interferogram mode, i.e. no onboard averaging. 


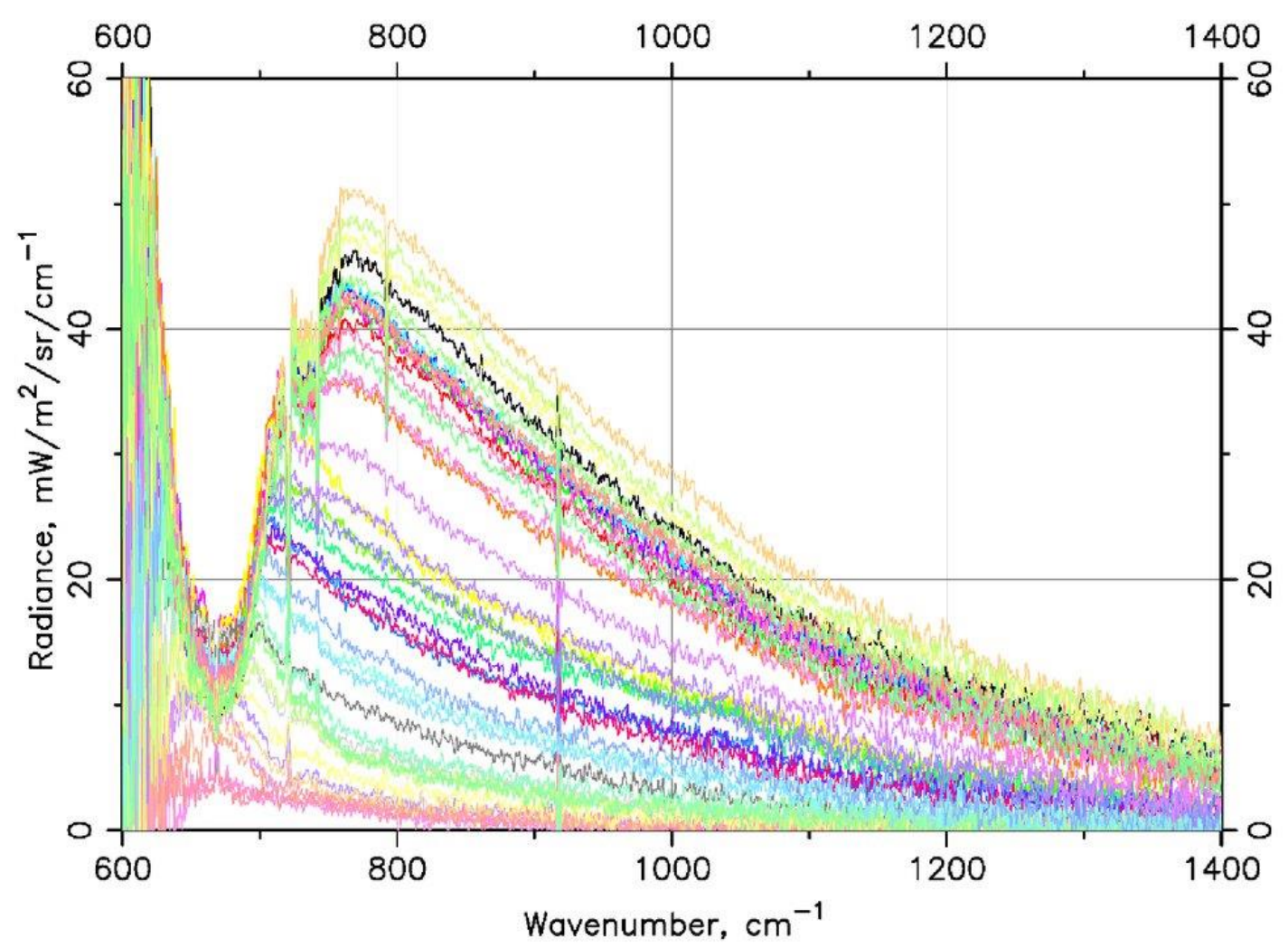

TIRVIM field-of-view center track on the surface of Mars and retrieved surface temperature obtained on March 15, 2018.

\section{Surface temperature $[K]$}

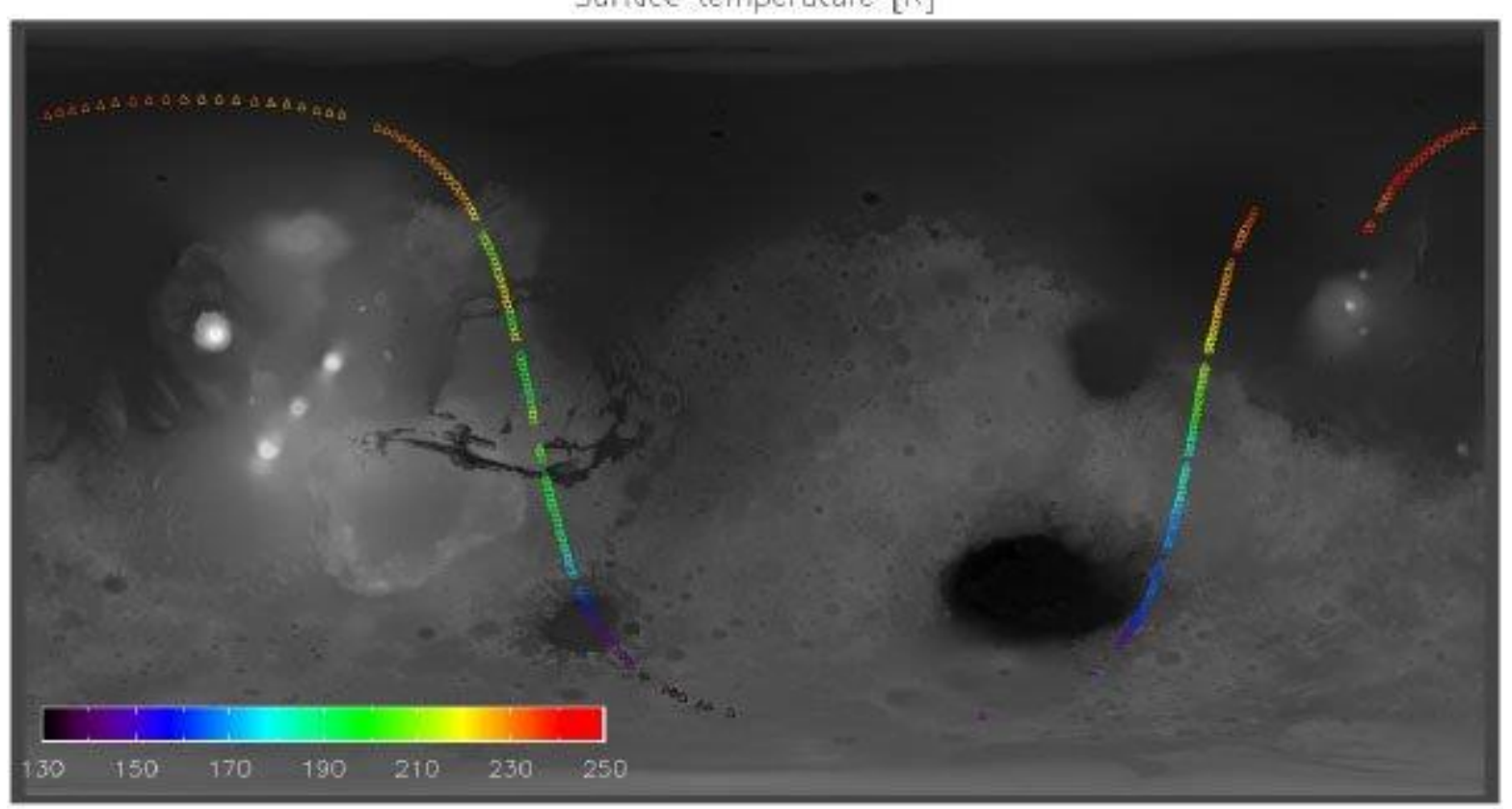

Temperature field along the TIRVIM field-of-view track on the surface measured obtained on March $15,2018$. 


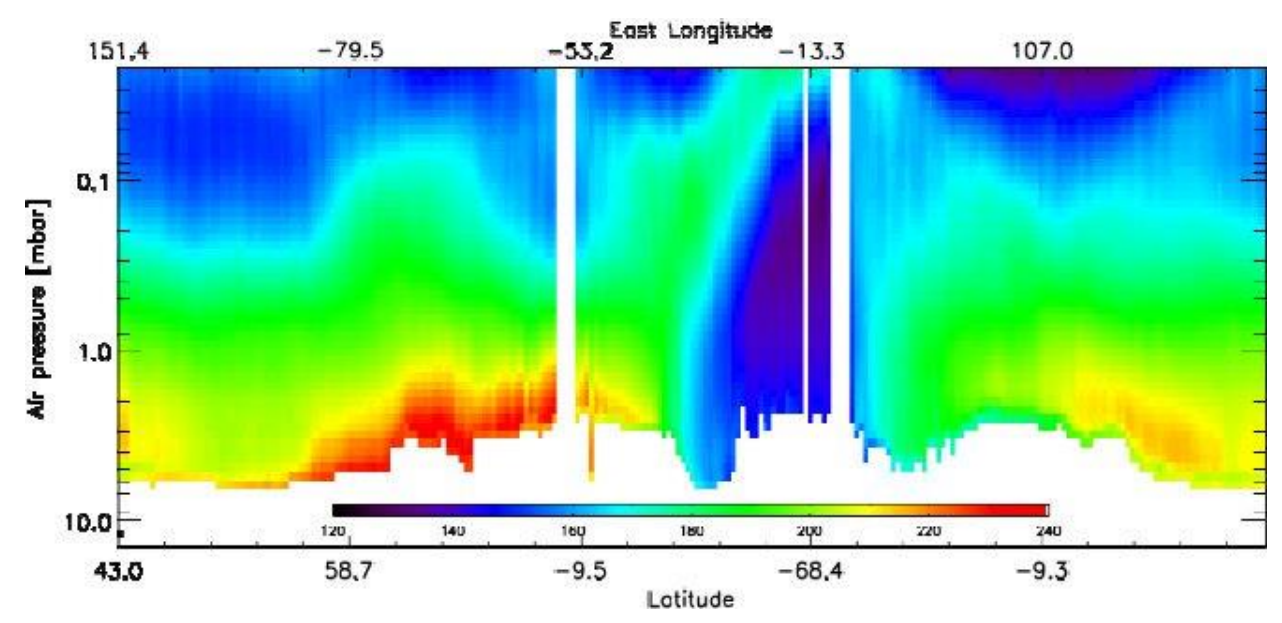

\section{AEROSOL PROPERTIES RETRIEVAL}

The main component of aerosols on Mars consists of mineral dust, while $\mathrm{H}_{2} \mathrm{O}$ ice and $\mathrm{CO}_{2}$ ice crystals also occur depending on the season and location. Wide spectral range of TIRVIM allows for spectral separation of dust and $\mathrm{H}_{2} \mathrm{O}$ ice particles.

Aerosol properties from TIRVIM solar occultation data are retrieved from 20 wavenumbers in the spectral range $1500-4500 \mathrm{~cm}^{-1}$ outside of strong gas absorption bands. The algorithm used for the preliminary retrievals of aerosol properties is straightforward and standard for all solar occultation experiments. The reference spectrum for an occultation $\mathrm{I}_{0}$ is obtained when observing the Sun out of the atmosphere. Inside the atmosphere solar radiance $I$ is attenuated by extinction of aerosols integrated over the line of sight $\mathrm{L}$. The ratio of this flux to the reference spectrum defines a spectrum of atmospheric transmission at a target altitude: $\mathrm{T}_{\mathrm{V}}(\mathrm{L})=\mathrm{I}_{\mathrm{V}}(\mathrm{L}) / \mathrm{I}_{0}$. Slant optical depth of aerosols is calculated from occultation techniques as $\tau_{\mathrm{v}}(\mathrm{L})=-\ln (\mathrm{T}(\mathrm{L}))$.

The procedure for the retrieval of the extinction vertical profiles is identical to the one described for SPICAM solar occultations on Mars [16, 17]; that is by making use of the standard 'onion peeling' method. Example of retrieved vertical profiles of transmission, slant opacity, and extinction coefficient for orbit \#9FC (recorded on 20 June 2018, latitude = 65N, Ls = 197, egress) are presented in Fig. 6 . Analysis of the spectral dependance of extinction coefficient recorded from this orbit reveals presence of two types of aerosols: dust particles at altitudes $10-18 \mathrm{~km}$ and water ice crystalls at $20-30 \mathrm{~km}$. Further steps, involving modelling of extinction coefficient spectral dependance and its fitting to the experimental extinction, provide information on vertical size distribution of the aerosols.

Retrieved vertical profiles of transmission (a), slant opacity (b), and extinction coefficient (c) from orbit \#9FC for 20 wavenumbers in 1500-4500 cm-1 (egress). 


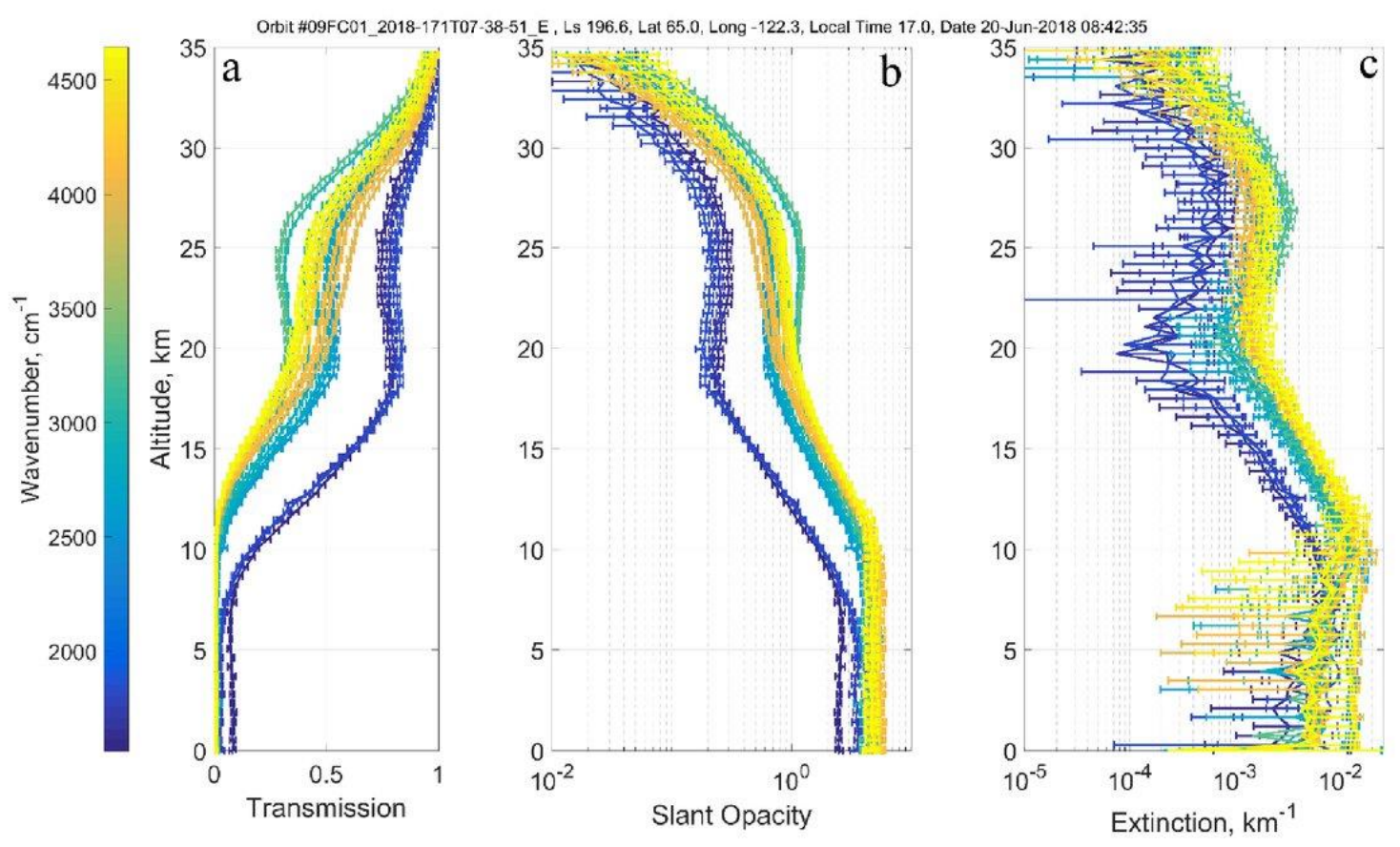

\section{CONCLUSIONS}

ACS TIRVIM FTS is aimed on long term monitoring of the thermal field and aerosol properties in the Martian atmosphere. The calibrations data (inflight and ground based) and results of the first measurements from the nominal science orbit show high instrument performance.

\section{ACKNOWLEDGEMENTS}

ExoMars is a space mission by ESA and Roscosmos. The development of TIRVIM funded by Roscosmos. Alexey Shakun, Boris Moshkin, and Oleg Korablev acknowledge support from Russian Science Foundation (RSF grant 16-12-10453) allowing the calibration of TIRVIM black body (Section 2). Other coauthors from IKI acknowledge FANO funding under contract PLANETA 0028-20140004. Work of Davide Grassi is supported by Italian Space Agency (ASI) through contract 2018-2HH.0 "Nomad".

\section{REFERENCES}

R.W. Zurek, A. Chicarro, M.A. Allen, J.-L. Bertaux, R.T. Clancy, F. Daerden, V. Formisano, J.B. Garvin, G. Neukum, M.D. Smith, Assessment of a 2016 mission concept: the search for trace gases in the atmosphere of Mars. Planet. Space Sci. 59, 284-291 (2011). https://doi.org/10.1016/j.pss.2010.07.007.

O. Korablev, F. Montmessin, A. Trokhimovskiy, A.A. Fedorova, A.V. Shakun, A.V. Grigoriev, B.E. Moshkin, N.I. Ignatiev, et al., "The Atmospheric Chemistry Suite (ACS) of three spectrometers for the ExoMars 2016 Trace Gas Orbiter," Space Sci Rev, 214:7, doi: https://doi.org/10.1007/s11214-0170437-6 (2018).

O. Korablev, A. Trokhimovsky, A. V. Grigoriev, A. Shakun, Y. S. Ivanov, B. Moshkin, K. Anufreychik, D. Timonin, I. Dziuban, Y. K. Kalinnikov and F. Montmessin, "Three infrared 
spectrometers, an atmospheric chemistry suite for the ExoMars 2016 trace gas orbiter," J. Appl. Remote Sens. 8(1), id. 084983 (2014). https://doi.org/10.1117/1.JRS.8.084983.

Bell, R.J., "Introductory Fourier transform spectroscopy", Academic Press, New York and London, 1972.

Hanel, R. A. B. Schlachman, F. D. Clark, C. H. Prokesh, J. B. Taylor, W. M. Wilson, and L. Chaney, "The Nimbus III Michelson Interferometer," Appl. Opt. 9, 1767 (1970). https://doi.org/10.1364/AO.9.001767.

Oertel, D., Moroz, V. I., Nopirakovskii, I., Linkin, V. M., Jahn, H., Kremnev, R. S., Becker-Ross, H., Stadthaus, W., Kerzhanovich, V. V., Matsygorin, I. A., D’yachkov, A. V. Khlyustova, L. I., Berwald, W. Ulich, M., Dreischer, H., Skrbek, W., Studemund, H., Schuster, R., Kaiser, G., Ignatova, S. P., Zelenov, I. A., Tserenin, I. D., Spankuch, D., Dohler, W., Schafer, K., Zasova, L. V., Ustinov, E. A., Fellberg, G., Lipatov, A. N., Shurupov, A. A., and Khavenson, N. G., "VENERA-15 and VENERA16 Infrared Experiment. Technique and First Results," Cosmic Research 23(, 62-175 (1985).

Christensen, P. R., J. L. Bandfield, V. E. Hamilton, S. W. Ruff, H. H. Kieffer, T. N. Titus, M. C. Malin, R. V. Morris, M. D. Lane, R. L. Clark, B. M. Jakosky, M. T. Mellon, J. C. Pearl, B. J. Conrath, M. D. Smith, R. T. Clancy, R. O. Kuzmin, T. Roush, G. L. Mehall, N. Gorelick, K. Bender, K. Murray, S. Dason, E. Greene, S. Silverman and M. Greenfield, "Mars Global Surveyor Thermal Emission Spectrometer experiment: Investigation description and surface science results," Journal of Geophysical Research 106, 23823-23872 (2001) https://doi.org/10.1029/2000JE001370.

Giuranna,M., V. Formisano, D. Biondi, A. Ekonomov, S. Fonti, D. Grassi, H. Hirsch, I. Khatuntsev, N. Ignatiev,b, M. Malgoska, A. Mattana, A. Maturilli, E. Mencarelli, F. Nespoli, R. Orfei, P. Orleanski, G. Piccioni, M. Rataj, B. Saggin, L. Zasova, Calibration of the Planetary Fourier Spectrometer longwavelength channel, Planet. Space Sci. 53, 993-1007 (2005). https://doi.org/10.1016/j.pss.2005.02.007.

Revercomb, H. E., H. Buijs, Hugh B. Howell, D. D. LaPorte, William L. Smith, and L. A. Sromovsky. Radiometric calibration of IR Fourier transform spectrometers: solution to a problem with the HighResolution Interferometer Sounder. Applied Optics 27, 3210 (1988). https://doi.org/10.1364/AO.27.003210.

Hanel, R., Conrath, B., Hovis, W., Kunde, V., Lowman, P., Maguire, W., Pearl, J., Pirraglia, J., Prabhakara, C., and Schlachman, B., Investigation of the Martian environment by infrared spectroscopy on Mariner 9. Icarus 17, 423-442 (1972). https://doi.org/10.1016/0019-1035(72)900097.

Smith, M. D., J. C. Pearl, B. J. Conrath and P. R. Christensen, "One Martian year of atmospheric observations by the thermal emission spectrometer," Geophysical Research Letters 28, 4263-4266 (2001) https://doi.org/10.1029/2001GL013608. 
Smith, M. D., "Interannual variability in TES atmospheric observations of Mars during 1999-2003," Icarus 167, 148-165 (2004). https://doi.org/10.1016/j.icarus.2003.09.010.

Giuranna, M.; Wolkenberg, P.; Grassi, D.; Aronica, A.; Aoki, S.; Formisano, V.; Scaccabarozzi, D.; Saggin, B., 12 Years of Atmospheric Monitoring by the Planetary Fourier Spectrometer onboard Mars Express. The Sixth International Workshop on the Mars Atmosphere: Modelling and observation was held on January 17-20 2017, in Granada, Spain, p. 1203.

Wolkenberg, P.; Giuranna, M.; Grassi, D.; Aronica, A.; Aoki, S.; Scaccabarozzi, D.; Saggin, B., Characterization of dust activity on Mars from MY27 to MY32 by PFS-MEX observations. Icarus 310, 32-47. .https://doi.org/10.1016/j.icarus.2017.10.045.

Grassi, D., Ignatiev, N. I., Zasova, L. V.; Maturilli, A., Formisano, V., Bianchini, G. A., Giuranna, M. (2005). Methods for the analysis of data from the Planetary Fourier Spectrometer on the Mars Express Mission. Planet. Space. Sci., v. 53, no. 10, p. 1017-1034. https://doi.org/10.1016/j.pss.2005.01.006.

Fedorova, A. A., Korablev, O. I., Bertaux, J.-L., Rodin, A. V., Montmessin, F., Belyaev, D. A., \& Reberac, A., "Solar infrared occultation observations by SPICAM experiment on Mars-Express: Simultaneous measurements of the vertical distributions of $\mathrm{H} 2 \mathrm{O}, \mathrm{CO} 2$ and aerosol," Icarus, 200(1), 96-117 (2009). https://doi.org/10.1016/j.icarus.2008.11.006.

Fedorova, A. A., Montmessin, F., Rodin, A. V., Korablev, O. I., Määttänen, A., Maltagliati, L., \& Bertaux, J.-L. "Evidence for a bimodal size distribution for the suspended aerosol particles on Mars," Icarus, 231, 239-260, (2014). https://doi.org/10.1016/j.icarus.2013.12.015.

(C) (2018) COPYRIGHT Society of Photo-Optical Instrumentation Engineers (SPIE). Downloading of the abstract is permitted for personal use only.

\section{Citation Download Citation}

Alexey Shakun, Alexey Shakun, Nikolay Ignatiev, Nikolay Ignatiev, Michail Luginin, Michail Luginin, Alexey Grigoriev, Alexey Grigoriev, Boris Moshkin, Boris Moshkin, Davide Grassi, Davide Grassi, Gabriele Arnold, Gabriele Arnold, Alessandro Maturilli, Alessandro Maturilli, Andrey Kungurov, Andrey Kungurov, Vladislav Makarov, Vladislav Makarov, Fedor Martynovich, Fedor Martynovich, Igor Maslov, Igor Maslov, Dmitry Merzlyakov, Dmitry Merzlyakov, Yuri Nikolskiy, Yuri Nikolskiy, Dmitry Patsaev, Dmitry Patsaev, Aleksandr Santos-Skripko, Aleksandr SantosSkripko, Oleg Sazonov, Oleg Sazonov, Viktor Shashkin, Viktor Shashkin, Igor Stupin, Igor Stupin, Alexander Zharkov, Alexander Zharkov, Oleg Korablev, Oleg Korablev, "ACS/TIRVIM: Calibration and first results", Proc. SPIE 10765, Infrared Remote Sensing and Instrumentation XXVI, 107650E (18 September 2018); doi: 10.1117/12.2322163; https://doi.org/10.1117/12.2322163 